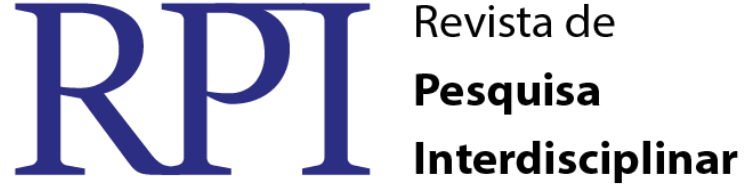

\section{BANCO INTERNACIONAL DE OBJETOS EDUCACIONAIS: CARACTERIZAÇÃO DOS OBJETOS VIRTUAIS DE APRENDIZAGEM DISPONIBILIZADOS PARA DOCÊNCIA EM QUÍMICA ANALÍTICA}

\author{
Egle Katarinne Souza da Silva - UFCG/CFP/UACEN \\ Luislândia Vieira de Figueiredo - UFCG/CFP/UACEN \\ Edilson Leite da Silva - UFCG/CFP/UACEN
}

\begin{abstract}
RESUMO
Atualmente estamos inseridos em uma sociedade globalizada, que passa por mudanças constantes e de forma acelerada. Diante dessa realidade de evolução tecnológica, as informações são processadas em questão de segundos e os recursos tecnológicos podem ser utilizados em todas as áreas do conhecimento assumindo um papel de auxiliador na construção do conhecimento. No contexto educacional são diversas ferramentas como as colaborativas, sistemas de busca, base de dados, objetos de aprendizagem, jogos educativos, redes sociais, dentre outras, algumas criadas já com o propósito de, ou adaptadas para, auxiliar no processo de ensino aprendizagem. Dentre estas ferramentas, os Objetos Virtuais de Aprendizagem são recursos digitais importantes para processo de ensino aprendizagem, que podem ser reutilizados e combinados com outros objetos para formar um ambiente de aprendizado rico e flexível. Partindo deste pressuposto, esta pesquisa objetiva apresentar para docentes e discentes estes objetos disponibilizados no Banco Internacional de Objetos de Aprendizagem que podem auxiliar no Ensino Superior de Química, especificamente no componente curricular Química Analítica. Trata-se de uma pesquisa bibliográfica, com abordagem quantitativa e qualitativa, também adota procedimento metodológico de um estudo de caso, pois a pesquisa centrouse numa particularidade específica. A coleta de dados foi realizada diretamente na página do banco de dados, sendo os resultados expostos em figuras e quadros e analisados de forma descritiva e exploratória. Percebe-se que estão disponíveis 33 objetos para Química Analítica, divididos em categorias conforme a organização do banco de dados, que são animações/simulações, áudios, experimentos práticos, hipertextos, imagens, mapas, softwares educativos e vídeos, que podem ser utilizados diretamente via internet ou serem salvos e usados em outra ocasião.
\end{abstract}

Palavras chave: Objetos Virtuais de Aprendizagem, Banco Internacional de Objetos Educacionais, Química Analítica.

\section{BANK INTERNATIONAL EDUCATIONAL OBJECTS: CHARACTERIZATION OF VIRTUAL LEARNING OBJECTS AVAILABLE FOR ANALYTICAL CHEMISTRY TEACHING.}

\begin{abstract}
Many people are inserted in a global society that is constantly changing and accelerated manner. Given this reality of technological development, information is processed in seconds and technological resources can be used in all knowledge areas assuming a supportive role in the construction of knowledge. There are several tools, in educational context, created for the purpose of (or adapted to) assist in the teaching and learning process as such collaborative, search engines, databases, learning objects, educational games, social networks. Among these tools, the Virtual Learning Objects (OVAs) are important digital resources for teaching and learning process, which can
\end{abstract}


be reused and combined with other objects to create a rich and flexible learning environment. This article aims to present these objects, available in the International Bank Learning Objects, to teachers and students for Chemistry Higher Education, specifically of Analytical Chemistry classes. This paper is a bibliographical, quantitative and qualitative research and it is also defined as a case study because the research focused on a specific feature. Data collection was performed directly on the database page and the results are presented in figures and tables, and analyzed in a descriptive and exploratory method. The results show that 33 virtual objects are available for curriculum component Analytical Chemistry and they are divided into categories according to the organization's database as such animations / simulations, audio, practical experiments, hypertext, images, maps, educational softwares and videos, which can be used directly via the Internet or be saved and used on another occasion.

Keywords: Virtual Learning Objects, International Bank of Educational Objects, Analytical Chemistry.

\section{INTRODUÇÃO}

Atualmente estamos inseridos em uma sociedade globalizada, que passa por mudanças constantes e de forma acelerada. Estamos perante uma nova realidade, a revolução tecnológica, onde as informações são processadas em questão de segundos. Neste contexto todos os parâmetros que compõe a sociedade atual: a saúde, segurança, economia, educação, etc. se voltam para esse processo de globalização.

A utilização da tecnologia aliada à educação contribui significativamente para construção do conhecimento. Sendo o uso dos computadores e outras tecnologias digitais uma forma dos professores auxiliarem os alunos no processo de ensino aprendizagem para facilitar na construção do conhecimento. Valente (1993) afirma que o computador pode provocar uma mudança no paradigma pedagógico, sendo usado na educação como máquina de ensinar ou como ferramenta.

O uso de computadores no ambiente escolar serve como uma ponte de aproximação entre aluno-conhecimento-professor, onde juntos de forma prazerosa e menos monótona constroem o conhecimento. Porém para que a aprendizagem seja eficiente com a utilização de computadores, é necessário que os professores estejam adequadamente preparados, tenham um conhecimento aprofundado sobre as tecnologias e/ou os recursos disponibilizados e, que atuam como facilitadores no processo de ensino e aprendizagem. Conforme afirma Kenski (2008 p. 77):

RPI Revista de Pesquisa Interdisciplinar, Cajazeiras, v. 1, Ed. Especial, 191 - 201, set/dez. de 2016. 
É necessário que os professores se sintam confortáveis para utilizar esses novos auxiliares didáticos. Estar confortável significa conhecê-los, dominar os principais procedimentos técnicos para sua utilização, avaliá-los criticamente e criar novas possibilidades pedagógicas, partindo da integração desses meios com o processo de ensino.

Partindo do pressuposto que a maior dificuldade encontrada pelos professores atualmente é a construção de uma aprendizagem significativa onde os alunos sejam agentes ativos contribuintes para a construção de seu próprio conhecimento, desenvolveu-se esta pesquisa que objetiva apresentar aos docentes e discentes os Objetos Virtuais de Aprendizagem (OVA) disponibilizados pelo Banco Internacional de Objetos Educacionais (BIOE) para a disciplina de Química Analítica.

\section{Referencial Teórico}

A utilização de recursos digitais no ambiente escolar contribui de forma significativa para o ensino, agilizando a comunicação entre professores, alunos e instituições. Nesse contexto os recursos digitais assumem o papel de facilitador para construção do conhecimento, podendo ser entre outros, um software específico para cada disciplina.

Existem vários softwares educativos que podem auxiliar no processo de ensino aprendizagem, um exemplo são os OVAs, que podem ser definidos segundo Tarouco; Fabre; Tamusiunas (2003, p. 02) "como qualquer recurso, suplementar ao processo de aprendizagem, que pode ser reusado para apoiar a aprendizagem”. Esses objetos aproximam o alunado dos conceitos e contribuem de forma prática na assimilação dos conteúdos em todas as áreas do conhecimento.

Os OVAs são recursos digitais importantes para processo de ensino aprendizagem, que podem ser reutilizados e combinados com outros objetos para formar um ambiente de aprendizado rico e flexível. Sendo assim Spinelli (2007, p. 7) diz que:

Um objeto virtual de aprendizagem é um recurso digital reutilizável que auxilia na aprendizagem de algum conceito e, ao mesmo tempo, estimula o desenvolvimento de capacidades pessoais, como por exemplo, imaginação e criatividade. Dessa forma, um objeto virtual de aprendizagem pode tanto contemplar um único conceito quanto englobar todo o corpo de uma teoria. Pode ainda compor um percurso didático, envolvendo um conjunto de

RPI Revista de Pesquisa Interdisciplinar, Cajazeiras, v. 1, Ed. Especial, 191 - 201, set/dez. de 2016. 
atividades, focalizando apenas determinado aspecto do conteúdo envolvido, ou formando, com exclusividade, a metodologia adotada para determinado trabalho.

Os OVAs são disponibilizados em vários portais, como: o projeto RIVED (Rede Interativa Virtual de Educação), disponível no endereço http://rived.mec.gov.br/; o LabVirt (Laboratório Didático Virtual) da USP, disponível em http://labvirt.fe.usp.br/ funciona como um repositório de simulações computacionais explorando os conteúdos de Física e Química; o Banco Internacional de Objetos Educacionais (BIOE), encontrado em http://objetoseducacionais2.mec.gov.br/.

Tanto o RIVED como o BIOE estão ligados ao Ministério de Educação (MEC), entretanto o BIOE é um dos repositórios mais completo e bem organizado. Silva e Salviano (2015, p. 03) afirma que:

Neste repositório existem objetos de diferentes países e línguas, permitindo a qualquer professor, de qualquer parte do mundo, acessar, utilizar e submeter os recursos em sua língua materna, publicando as suas produções em um processo colaborativo.

Segundo Afonso (2010) o BIOE foi criado em 2008 pelo Ministério da Educação, em parceria com o Ministério da Ciência e Tecnologia, Rede Latinoamericana de Portais Educacionais - RELPE, Organização dos Estados Ibero-americanos - OEI e outros.

No BIOE, os OVAs encontram-se divididos por níveis de ensino que compreendem os padrões determinados pela Lei de diretrizes e Bases (LDB), são eles: Educação Infantil, Ensino Fundamental, Ensino Médio, Educação Profissional, Educação Superior e Modalidades de Ensino, sendo esta subdividida em Educação de Jovens e Adultos (EJA) e Educação Escolar Indígena. Para cada nível de ensino existem objetos virtuais de aprendizagem que contemplam seus respectivos componentes curriculares. Para todos os componentes curriculares são disponibilizadas 08 categorias, que são: Animação/Simulação, Áudio, Experimento Prático, Hipertexto, Imagem, Mapa, Software Educacional e Vídeo.

Para o Ensino Superior o BIOE oferece diversos objetos que compreende determinadas áreas do conhecimento, dentre elas destaca-se a Área de Ciências Exatas e da Terra, na qual encontra-se o curso de química, que possui o componente curricular Química Analítica.

RPI Revista de Pesquisa Interdisciplinar, Cajazeiras, v. 1, Ed. Especial, 191 - 201, set/dez. de 2016. 
Muitos compostos químicos precisam ser identificados, para isto é necessário um estudo detalhado, com etapas bem definidas. O estudo para identificação dos constituintes bem como a quantidade destes pode ser feita através da análise química. Vogel (2013, p.01) afirma que:

\begin{abstract}
A análise química é definida como a aplicação de um processo ou de uma série de processos para identificar ou quantificar uma substância, ou os componentes de uma solução ou mistura ou, ainda, para determinar as estruturas de compostos químicos.
\end{abstract}

A análise química se divide em duas áreas: A Química Analítica Qualitativa e a Química Analítica Quantitativa.

A Química Analítica Qualitativa se preocupa com a identificação dos constituintes de uma amostra, seja ela de natureza mineral, vegetal ou animal. Segundo Vaitsman e Bittencourt (1995, p.01) “o procedimento para a identificação de uma espécie química consiste em provocar, na mesma, uma variação em suas propriedades, que possa ser facilmente observada e que corresponda com a constituição da dita substância."

Para Baccan et al (1995) a Química Analítica Quantitativa é utilizada para determinar as concentrações, volumes ou massa exata dos componentes presentes na amostra, através das técnicas de gravimetria, volumetria, entre outras. Dessa maneira a química analítica quantitativa está relacionada com a separação, identificação e determinação das quantidades relativas dos componentes de uma amostra.

\title{
Metodologia
}

Desenvolveu-se esta pesquisa na preocupação de identificar a quantidade e qualidade dos objetos virtuais de aprendizagem disponíveis no BIOE para o Ensino Superior de Química, da disciplina de Química Analítica.

Inicialmente realizou-se uma pesquisa bibliográfica, a fim de coletar em artigos e periódicos as definições que norteiam a temática abordada. Trata-se de uma pesquisa quantitativa, pois apresenta as quantidades de objetos de aprendizagem disponíveis no BIOE para a disciplina de Química Analítica, bem como qualitativa no sentido de analisar a 
qualidade dos recursos oferecidos e identificar as possíveis carências do repositório relacionada a esse contexto.

Para Mattar (1993) as pesquisas bibliográficas é uma forma prática e econômica de aprofundar um problema de pesquisa embasado por trabalhos que já foram elaborados anteriormente. Nessa perspectiva a fonte utilizada para realização deste tipo de pesquisa consiste em livros, periódicos, teses, dissertações, banco ou base de dados, etc.

A pesquisa qualitativa segundo Bogdan \& Biklen (2003), envolve a obtenção de dados descritivos, obtidos no contato direto do pesquisador com a situação estudada, prioriza mais o processo do que o produto.

Para Mattar (2001), a pesquisa quantitativa busca validar as hipóteses mediante ao uso de dados estruturados, estatísticos, com análise de um número de casos representativos, recomendando um curso final da ação. Dessa maneira, a característica principal da pesquisa quantitativa é quantificar os dados e generalizar os resultados da amostra para os interessados.

Como o foco da pesquisa se concentrou em uma temática especifica, define-se também como um estudo de caso. Conforme Prodanov e Freitas (2013, p. 60) "o estudo de caso abrange o estudo intenso e cansativo de um ou poucos objetos de maneira que permita o seu amplo e detalhado conhecimento". Dessa forma o estudo de caso pode ser utilizado quando pretende-se alcançar resultados relevantes diante de uma situação pré-determinada, como forma de investigação aprofundada.

\section{Resultados}

Na Figura 1 observa-se a página inicial do BIOE, como este repositório é organizado de maneira sistemática, os recursos digitais oferecidos encontram-se divididos por níveis de ensino. A figura representa a tela do repositório depois dos seguintes passos: Clicou-se no nível de Ensino Superior (Nova Estrutura), posteriormente escolheu-se a opção Ciências Exatas e da Terra onde se encontra os objetos virtuais de aprendizagem para o curso de Química. Diante da figura exposta percebe-se a subdivisão dos níveis de ensino em 08 categorias, são elas: Animação/Simulação, Áudio, Experimento Prático, Hipertexto, Imagem, Mapa, Software Educacional e Vídeo.

A área de Ciências Exatas e da Terra disponibiliza 3321 objetos que são divididos de acordo com os seguintes cursos superiores: Astronomia (332), Ciência da Computação (07), RPI Revista de Pesquisa Interdisciplinar, Cajazeiras, v. 1, Ed. Especial, 191 - 201, set/dez. de 2016. 
Física (1074), GeoCiências (70), Matemática (1414), Oceanografia (32), Probabilidade e Estatística (22) e Química (370). Observa-se que são oferecidos mais objetos para os cursos de Matemática e Física, juntas correspondem a 74,9\% dos objetos. O curso de Química com 370 objetos representa $11,1 \%$ dos OVAs.

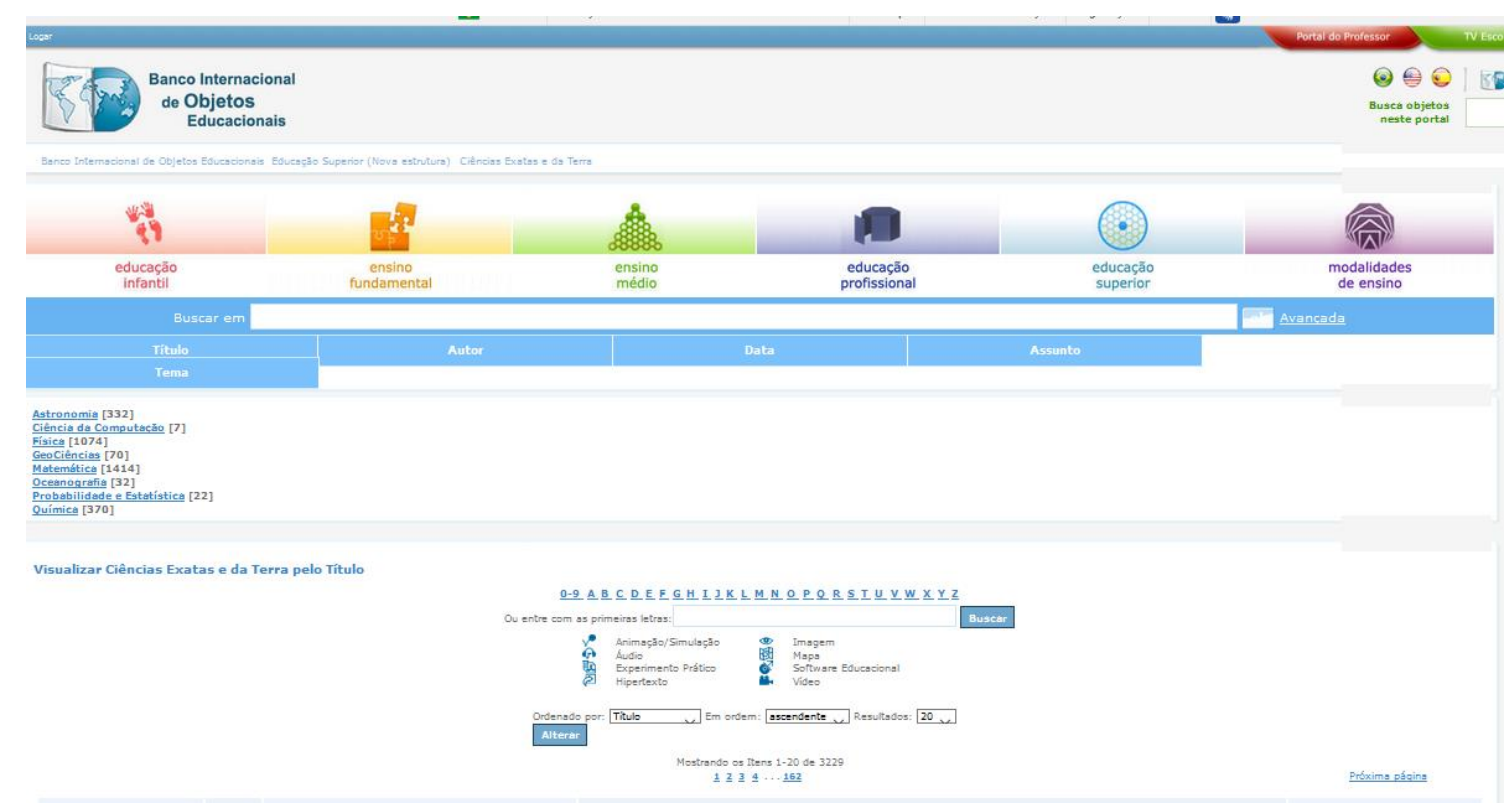

Fonte: BIOE. Acesso em: 12 de agosto de 2016

Figura1: Objetos para o Ensino Superior de Ciências Exatas e da Terra do BIOE.

Na Figura2 observa-se a divisão dos 370 OVAs disponíveis para o Ensino Superior de Química, por categorias, sendo: Animação/Simulação (57), Áudio (18), Experimento Prático (30), Hipertexto (0), Imagem (127), Mapa (0), Software Educacional (24) e Vídeo (114). Percebe-se que nas categorias Hipertexto e Mapa não existe nenhum objeto, em contra partida as categorias Imagens e Vídeo representam $65,1 \%$ desses objetos.

RPI Revista de Pesquisa Interdisciplinar, Cajazeiras, v. 1, Ed. Especial, 191 - 201, set/dez. de 2016. 


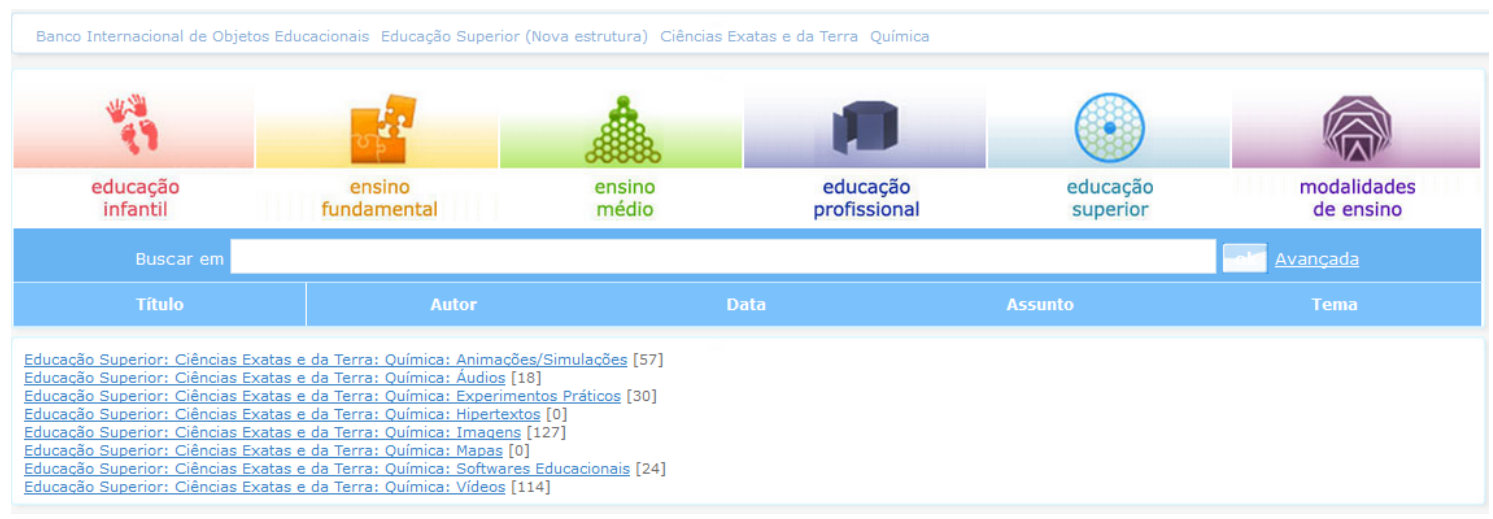

Fonte: BIOE. Acesso em: 12 de agosto de 2016

Figura 2: Objetos para o Ensino Superior de Química

Para identificação dos objetos virtuais de aprendizagem, fez-se uma pesquisa detalhada no BIOE por categoria. Conforme mostra a Quadro 1, são disponibilizados 33 OVAs para a disciplina de Química Analítica, que também é abordada no repositório pelo termo Instrumentação Analítica. Pode-se observar uma diversidade significativa relacionada tanto aos idiomas quanto aos tipos de recursos, porém os recursos Animação/Simulação e Áudios só oferecem um objeto cada, este dado demonstra uma carência do repositório. Percebe-se também que dois grupos de autores se destacam na produção destes OVAs: Lopez, Miguel A.F.; Barrio, Manuel G.; Pujato, Carmen C. L.; (na produção de imagens) e Sousa, Teresa (na produção de vídeos).

Quadro 1: Relaciona os OVAs com seus Autores, Componentes Curriculares e Tipo de Recurso 


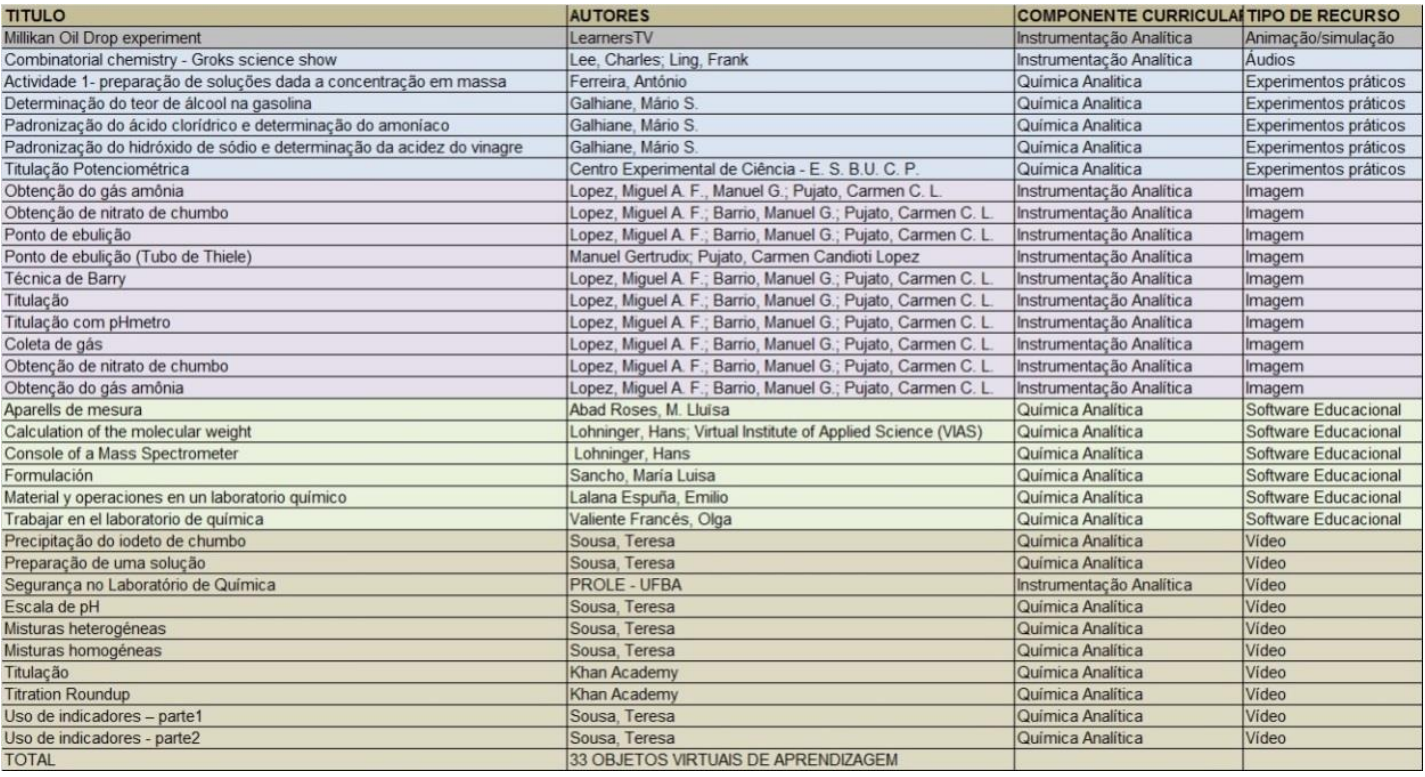

Fonte: BIOE. Acesso em: 17 de agosto de 2016

Com a finalidade de auxiliar os interessados em acessar os OVAs disponibilizados pelo BIOE para a disciplina de Química Analítica criou-se um quadro relacionando o título do objeto com seu respectivo objetivo.

Desta maneira, no Quadro 2, os interessados dispõem de um resumo detalhado, dos objetivos propostos pelos autores referentes a cada recurso, podendo facilitar a pesquisa de acordo com o que eles pretendam executar dentro da disciplina trabalhada. Exemplificando o Experimento Prático: Padronização do Hidróxido de Sódio e determinação da Acidez do Vinagre, objetiva definir propriedades de um ácido e de uma base, bem como, auxiliar o professor a explicar aos alunos o que determina a acidez e a basicidade de uma substância. 
Quadro 2: Relaciona os OVA com os Objetivos.

\begin{tabular}{|c|c|}
\hline TITULO & OBJETIVO DO OBJETO VIRTUAL DE APRENDIZAGEM \\
\hline Millikan Oil Drop experiment & To simulate the fall experiment of Robert Millikan, that illustrates the mesure of the eletron's electric charge \\
\hline Combinatorial chemistry - Groks science show & Show the development of a new field of the chemistry: the combinatorial chemistry \\
\hline Actividade 1- preparação de soluções dada a concentração em masse & Preparar soluções a partir de uma certa concentração em massa. \\
\hline Determinaccão do teor de álcool na gasolina & Determinar o teor e álcool na gasolina. \\
\hline Padronização do ácido cloridrico e determinação do amoniaco & $\begin{array}{l}\text { Ensinar a definição de ácido e de base e suas respectivas propriedades, o que determina a acidez ou basicidade de uma } \\
\text { substância aquosa, ensinar sobre reação de neutralizacăo ácido-base }\end{array}$ \\
\hline Padronização do hidróxido de sódio e determinação da acidez do vina & $\begin{array}{l}\text { Definir propriedades de um ácido e de uma base, auxiliar o professor a explicar aos alunos o que determina a acidez e a } \\
\text { basicidade de uma substância. }\end{array}$ \\
\hline Titulação Potenciométrica & Determinar a concentração de uma solução por potenciometria. \\
\hline Obtenção do gás amônia & Apresentar uma ilustração de aparelhagem para obtenção de amoniaco em laboratório. \\
\hline Obtenção de nitrato de chumbo & Apresentar um tipo de aparellagem para obtenção de nitrato de chumbo. \\
\hline Ponto de ebulição & Apresentar uma ilustração de aparelhagem para determinação de ponto de ebulição. \\
\hline $\begin{array}{l}\text { Ponto de ebulição (Tubo de Thiele) } \\
\end{array}$ & Apresentar o tubo de Thiele, uma vidraria de uso laboratorial. \\
\hline Técnica de Barry & Apresentar uma ilustração da técnica de Barry. \\
\hline Titulação & llustrar a aparelhagem para uma titulação, com bureta, suporte, garras e erlenmeyer. \\
\hline Titulação com pHmetro & Apresentar uma ilustração de uma aparelhagem para titulação. \\
\hline Coleta de gás & Apresentar um sistema de recolhimento de gás por deslocamento de água. \\
\hline Obtenção de nitrato de chumbo & Apresentar um tipo de aparelhagem para obtenção de nitrato de chumbo. \\
\hline Obtenção do gás amônia & Apresentar uma ilustração de aparelhagem para obtenção de amoniaco em laboratório. \\
\hline Aparells de mesura & Informar alguns dels dispositius de mesura en un laboratori de química, com ara els saldos, buretes, pipetes, termòmetres, etc \\
\hline Calculation of the molecular weight & Discover the weight or the molecular weight of substances from the isotopic composition of a particular molecule \\
\hline Console of a Mass Spectrometer & Help in understanding the observation of the chart obtained by the mass spectrometer of the compound 6-methyl-5-nonen-4-one \\
\hline Formulación & Informar sobre las fórmulas de la química inorgánica y sus compuestos \\
\hline Material y operaciones en un laboratorio quimico & Informar por medio de actividades cuales son los instrumentos utilizados en un laboratorio de química \\
\hline Trabajar en el laboratorio de química & Informar sobre el comportamiento correcto en laboratorios de química \\
\hline Precipitação do iodeto de chumbo & Mostrar a reação de nitrato de chumbo com iodeto de potássio formando um precipitado de cor amarela que é iodeto de chumbo. \\
\hline Preparação de uma solução & Ensinar técnicas de preparação de solução e utilização de vidraria de laboratório. \\
\hline Segurança no Laboratório de Química & Aprender sobre os cuidados ao manipular substâncias em um laboratório para evitar acidentes. \\
\hline Escala de pH & Explicitar sobre as propriedaces das substâncias e escala de Ph. \\
\hline Misturas heterogéneas & Estudar as misturas heterogêneas. \\
\hline Misturas homogéneas & Estudar as misturas homogéneas \\
\hline Titration Roundup & The aim of this educational object is to help students to understand titration curves \\
\hline Uso de indicadores - parte1 & Aiudar os alunos a compreender curvas de titulacão. \\
\hline Uso de indicadores - parte2 & Estudar as propriedades, características e diferenças das soluçōes ácidas, básicas e neutras. \\
\hline
\end{tabular}

Fonte:BIOE. Acesso em: 17 de agosto de 2016.

\section{Conclusão}

No ensino superior a inserção de novas tecnologias representam um dos fatores fundamentais no processo de ensino e aprendizagem, propiciando ao docente aquisição de habilidades com relação à busca de novas estratégias que viabilizem um aprendizado significativo em cada situação de ensino. Nesse contexto, o processo de ensino e aprendizagem, outorga aos docentes que adotam esses recursos como metodologia de ensino, um profissional com postura diferenciada.

A referente pesquisa demonstrou que o BIOE oferece 33 objetos virtuais de aprendizagem para a disciplina de Química Analítica, estes estão distribuídos em 04 idiomas e 06 categorias. Percebe-se que os objetos disponíveis apresentam boa qualidade, abrangendo vários conteúdos trabalhados em Química Analítica.

Foi possível detectar carências de objetos em algumas categorias Animação/Simulação (1) e Áudios (1) e Hipertexto (0) e Mapas (0), o que demonstra a necessidade de desenvolver mais objetos nestas categorias, voltados para os conteúdos de Química Analítica.

\section{REFERÊNCIAS Bibliográficas}

RPI Revista de Pesquisa Interdisciplinar, Cajazeiras, v. 1, Ed. Especial, 191 - 201, set/dez. de 2016. 
AFONSO, Maria da Conceição L. Banco Internacional de Objetos de Educacionais (BIOE): normas para definição dos metadados. Brasília: CESPE/UnB, MEC, 2010.

BACCAN, N.; et al. Química Analítica Quantitativa Elementar, 2.ed. Campinas: Editora da UNICAMP, 1995.

BOGDAN, R. S.; BIKEN, S. Investigação qualitativa em educação: uma INTRODUÇÃO à teoria e aos métodos. 12.ed. Porto: Porto, 2003.

MATTAR, F. N. - Pesquisa de Marketing - metodologia, planejamento, execução e análise. São Paulo: Atlas, 1993.

MATTAR, F. N. Pesquisa de marketing. 3.ed. São Paulo: Atlas, 2001.

KENSKI, Vani M. Tecnologias e Ensino Presencial e a Distância. Campinas, SP: Papirus, 2008

PRODANOV, Cleber Cristiano; FREITAS, Ernani Cesar de. Metodologia do trabalho científico: métodos e técnicas da pesquisa e do trabalho acadêmico. 2 ed. Novo Hamburgo:Freevale, 2013.

SABBATINI, Marcelo. Reflexões críticas sobre o conceito de objeto de aprendizagem aplicado ao ensino de ciências e matemática.Revista de Educação Matemática e

Tecnológica Iberoamericana - vol. 3 - número 3 - 2012.

SILVA, Edilson Leite da; SALVIANO, Adenilda Timóteo. Objetos de aprendizagem para o ensino de matemática. VIII Colóquio de Matemática IFCE- Juazeiro do Norte. 12 a 14 de agosto 2015.

SPINELLI, W. Os objetos virtuais de aprendizagem: ação, criação e conhecimento.2007. Disponível em: <http://www.lapef.fe.usp.br/rived/ textoscomplementares/textoImodulo5.pdf>. Acesso em:11 ago 2016.

TAROUCO, Liane Margarida Rockenbanch; FABRE, Marie-Cristine Julie Mascarenhas; TAMUSIUNAS, Fabrio Raupp. Reusabilidade de objetos educacionais. 2003. Disponível em: 〈http://seer.ufrgs.br/index.php/renote/ article/view/13628/7697>. Acesso em: 11 ago 2016.

VALENTE, J. A. Computadores e Conhecimento: repensando a educação. Por que o computador na educação. Gráfica central da Unicamp, Campinas, 1993.

VAITSMAN, Delmo S.; BITTENCOURT, Olymar A. Ensaios Químicos Qualitativos. Rio de Janeiro, Interciência, 1995.

VOGEL, A. I. Análise química quantitativa. 6. ed. Rio de Janeiro: LTC, 2002.

RPI Revista de Pesquisa Interdisciplinar, Cajazeiras, v. 1, Ed. Especial, 191 - 201, set/dez. de 2016. 